\title{
ACIDENTES OCUPACIONAIS COM A EQUIPE DE ENFERMAGEM DA ATENÇÃO HOSPITALAR*
}

Juliana Ladeira Garbaccio', Wiliam César Bento Regis², Rogério Magalhães Coimbra Silva ${ }^{3}$, Wilson Goulart Estevão ${ }^{4}$

${ }^{1}$ Enfermeira. Doutora em Enfermagem. Professora da Pontifícia Universidade Católica de Minas Gerais. Belo Horizonte-MG-Brasil. ${ }^{2}$ Biólogo. Doutor em Biologia. Professor da Pontifícia Universidade Católica de Minas Gerais. Belo Horizonte-MG-Brasil.

${ }^{3}$ Enfermeiro. Especialista em Saúde Pública. Divinópolis-MG-Brasil.

${ }^{4}$ Discente em Enfermagem. Pontifícia Universidade Católica de Minas Gerais. Arcos-MG-Brasil.

RESUMO: Este estudo objetivou descrever os acidentes ocupacionais com a equipe de enfermagem de dois hospitais públicos e suas notificações. Foi utilizada pesquisa transversal, realizada entre março e abril de 2012, na cidade de Arcos, no Centro-Oeste de Minas Gerais. Aplicou-se um questionário estruturado entre enfermeiros, técnicos e auxiliares de enfermagem. Os dados foram tabulados, analisados descritivamente e estatisticamente no programa Statistical Package for the Social Sciences, versão 13.0. O relato do índice de acidente de trabalho nas instituições, entre 2002 e 2011, foi de 59,6\%, dos quais 90\% de natureza biológica, 34\% não notificaram o acidente de trabalho. A faixa etária mais jovem (20-29 anos/81,8\%) e aqueles com menos tempo de experiência ( $\leq 5$ anos/84,2\%) relataram mais acidentes. Conclui-se que há pouca adesão pelos profissionais aos cuidados preconizados, verificando-se também a baixa porcentagem da Comunicação do Acidente do Trabalho, tornadose necessárias intervenções de educação e prevenção para minimizar os acidentes.

DESCRITORES: Saúde do trabalhador; Riscos ocupacionais; Acidentes de trabalho.

\section{OCCUPATIONAL ACCIDENTS WITH THE NURSING TEAM INVOLVED IN HOSPITAL CARE}

\section{ACCIDENTES OCUPACIONALES CON EL EQUIPO DE ENFERMERÍA DE LA ATENCIÓN HOSPITALAR}

RESUMEN: Este estudio tuvo la finalidad de describir los accidentes ocupacionales con el equipo de enfermería de dos hospitales públicos y sus notificaciones. Fue utilizada investigación transversal, realizada entre marzo y abril de 2012, en la ciudad de Arcos, Centro-Oeste de Minas Gerais. Fue utilizado un cuestionario estructurado entre enfermeros, técnicos y auxiliares de enfermería. Los datos fueron organizados, analizados descriptiva y estadísticamente en el programa Statistical Package for the Social Sciences, versión 13.0. El informe del índice de accidente de trabajo en las instituciones, entre 2002 y 2011, fue de 59,6\%, de los cuales $90 \%$ de naturaleza biológica, $34 \%$ no notificaron el accidente de trabajo. La franja etaria más joven (20-29 años/81,8\%) y aquellos con menos tiempo de experiencia ( $\leq 5$ años/84,2\%) relataron más accidentes. Se concluye que hay poca adhesión por los profesionales a los cuidados propuestos, verificándose también la baja porcentaje de la Comunicación del Accidente de Trabajo, siendo necesarias intervenciones de educación y prevención para minimizar los accidentes. DESCRIPTORES: Salud del trabajador; Riesgos ocupacionales; Accidentes de trabajo. accidents.

*Artigo extraído da monografia de conclusão do Curso de Enfermagem. Pontifícia Universidade Católica de Minas Gerais, 2013.

Pontifícia Universidade Católica de Minas Gerais

Rua N. S. do Livramento, 801 - 37925-000 - Piumhi-MG-Brasil

E-mail: goulartwilson@hotmail.com 


\section{INTRODUÇÃO}

A Enfermagem, como profissão, é dividida nas categorias de auxiliares de enfermagem, técnicos de enfermagem e enfermeiros. Na jornada de trabalho eles prestam assistência constante aos pacientes 24 horas por dia, executando cerca de $60 \%$ das ações de saúde, sendo os profissionais da saúde que mais entram em contato físico com os pacientes ${ }^{(1)}$.

Os trabalhadores da Enfermagem, durante a assistência ao paciente, estão expostos a numerosos riscos de fontes químicas, representados pela poeira, vapores, gases; fontes físicas que correspondem ao ruído, calor, frio, radiações ionizantes e não ionizantes, vibrações, umidade, pressões ambientais; fontes biológicas que se relacionam aos agentes biológicos, que entram em contato com o trabalhador pelo contato direto ou indireto (como os acidentes com material perfurocortante); fontes ergonômicas caracterizadas pelo transporte manual de cargas e peso, repetitividade, ritmo excessivo de trabalho, posturas inadequadas e riscos de acidentes que correspondem ao arranjo físico inadequado, quedas, equipamentos sem dispositivos de segurança ${ }^{(2)}$.

O contato próximo com pacientes portadores de diversas enfermidades e a realização de vários procedimentos assistenciais contribui para a exposição dos profissionais a agentes biológicos e químicos, além do desgaste psíquico. Desta forma, as condições de trabalho para a equipe de saúde nos hospitais têm sido consideradas insalubres por agrupar inúmeras situações de riscos ocupacionais $^{(3)}$.

As infraestruturas irregulares alheias à Norma Regulamentadora 32 do Ministério do Trabalho e Emprego do Brasil também são fatores cruciais ao risco ocupacional. Além de o hospital representar um local de risco para os seus trabalhadores, os próprios profissionais podem apresentar comportamentos de risco, pela execução de tarefas de modo inadequado, sem o uso de Equipamentos de Proteção Individual (EPI) e/ou ausência ou baixa capacitação na prevenção de acidentes ocupacionais ${ }^{(4)}$.

A CAT (Comunicação de Acidente de Trabalho) é o documento responsável pela notificação do acidente de trabalho, sendo preenchido pelo trabalhador. Essa comunicação é obrigatória pela Lei $n^{\circ} 8.213 / 91$, independentemente da gravidade, e encaminhada pela empresa à Previdência Social até o primeiro dia útil seguinte da ocorrência do acidente, mesmo que não haja afastamento do acidentado e, em caso de morte, de imediato à autoridade competente, sob pena de multa. Posteriormente a esse procedimento serão realizadas a avaliação médica e concessão do benefício ${ }^{(5-6)}$.

Levando em consideração o fato de os hospitais representarem um local de risco para seus trabalhadores e a equipe de enfermagem como aquela com maior chance de se envolver em acidentes laborais, surgiu a seguinte pergunta norteadora desta pesquisa: Qual foi a ocorrência de acidente de trabalho com os profissionais de enfermagem da atenção hospitalar de uma cidade do Centro-Oeste de Minas Gerais, no período de 2002 a 2011?

Neste sentido, esta pesquisa teve como objetivo descrever os acidentes ocupacionais e suas notificações entre a equipe de enfermagem de dois hospitais públicos.

\section{MÉTODO}

Tratou-se de uma pesquisa transversal, conduzida no período entre março e abril de 2012, em dois hospitais públicos, na cidade de Arcos, no Centro-Oeste de Minas Gerais, com aproximadamente 36.000 habitantes, após aprovação do Comitê de Ética em Pesquisa da Pontifícia Universidade Católica de Minas Gerais (CAAE 07352212.1.0000.5137).

A coleta de dados foi realizada por meio de entrevistas com enfermeiros, técnicos e auxiliares de enfermagem que trabalhavam alocados em três turnos (manhã, tarde e noite, no período de 8 horas diárias e em escalas 12/36). As entrevistas ocorreram após o convite verbal aos profissionais, explicando os objetivos e relevância da pesquisa e, após a concordância, foi apresentado a eles o termo de consentimento livre e esclarecido. Foram excluídos da pesquisa aqueles que estavam de férias no período da coleta de dados.

Utilizou-se um impresso elaborado para a pesquisa contendo questões fechadas e abertas referentes aos dados sociodemográficos e aos acidentes de trabalho, bem como as notificações deles, no período de 2002 a 2011.

Os dois hospitais onde a pesquisa foi 
conduzida eram de pequeno porte, prestavam serviços gerais de clínica médica e cirúrgica, atendimento a pequenas urgências e emergências, com frequências similares.

Os dados obtidos foram tabulados, analisados descritiva e estatisticamente pelo programa estatístico Statistical Package for the Social Sciences, versão 13.0. A associação entre as variáveis sociodemográficas e a variável ocorrência de acidente de trabalho foi verificada pela análise bivariada tendo como testes o QuiQuadrado de Pearson, adotando-se nível de significância de 0,05 e o intervalo de confiança de $95 \%$.

\section{RESULTADOS}

As entrevistas foram realizadas com 57 profissionais e não houve recusas para participar da pesquisa. Os dados coletados em ambos os hospitais foram analisados em conjunto devido às semelhanças dos resultados e das características de cada instituição.

Verificou-se predomínio de entrevistados do sexo feminino $(91,2 \% / 52)$, faixa etária entre 30 e 39 anos (42,1\%/24), idade média de 35 anos, técnicos de enfermagem (72\%/41), até cinco anos de trabalho na categoria profissional (33,3\%/19). Demais dados sociodemográficos estão apresentados na Tabela 1.

Entre os 57 participantes, 34 (59,6\%) relataram ter sido acometidos por algum tipo de acidente de trabalho na instituição, havendo predominância nos profissionais mais jovens - 20-29 anos (81,8\%/9), técnicos de enfermagem $(68,3 \% / 28)$, com até cinco anos de trabalho na categoria $(84,2 \% / 16)$, no turno diurno $(73,5 \% / 25)$ (Tabela 1$)$.

Entre os profissionais que referiram acidentes, houve maior relato entre os homens $(80 \%)$, e, apesar de serem relatados acidentes em maior percentual entre homens, em quantidade numérica o acidente com as mulheres é sete vezes maior, sendo apresentada uma diferença absoluta de quatro masculinos contra 30 femininos. Ressalta-se que, além do número de técnicos de enfermagem ser numericamente maior que o de enfermeiros, é também mais relatado, percentualmente, acidentes nesta categoria.

Tabela 1 - Dados sociodemográficos da equipe de Enfermagem e acidentes ocupacionais. Arcos-MG, 2012

\begin{tabular}{|c|c|c|c|c|}
\hline \multirow{2}{*}{ Variáveis sociodemográficas } & \multicolumn{2}{|c|}{ Equipe de Enfermagem } & \multicolumn{2}{|c|}{ Profissionais que referiram acidentes } \\
\hline & $\mathrm{N}=57$ & $\%$ & $\mathrm{n}=34$ & $\%$ \\
\hline \multicolumn{5}{|l|}{ Sexo } \\
\hline Feminino & 52 & 91,2 & 30 & 57,7 \\
\hline Masculino & 5 & 8,8 & 4 & 80,0 \\
\hline \multicolumn{5}{|l|}{ Faixa etária (anos) } \\
\hline 20 a 29 & 11 & 19,3 & 9 & 81,8 \\
\hline 30 a 39 & 24 & 42,1 & 14 & 58,3 \\
\hline 40 a 49 & 15 & 26,3 & 10 & 66,7 \\
\hline 50 a 59 & 7 & 12,3 & 1 & 14,3 \\
\hline \multicolumn{5}{|l|}{ Categoria profissional } \\
\hline Técnico de enfermagem & 41 & 72 & 28 & 68,3 \\
\hline Enfermeiro & 8 & 14 & 2 & 25,0 \\
\hline Auxiliar de enfermagem & 8 & 14 & 4 & 50,0 \\
\hline \multicolumn{5}{|c|}{ Tempo de trabalho na categoria (anos) } \\
\hline$\leq 5$ & 19 & 33,3 & 16 & 84,2 \\
\hline 6 a 10 & 11 & 19,3 & 9 & 81,8 \\
\hline 11 a 15 & 14 & 24,6 & 5 & 35,7 \\
\hline$\geq 16$ & 13 & 22,8 & 4 & 30,8 \\
\hline \multicolumn{5}{|l|}{ Turno de trabalho } \\
\hline Diurno & 34 & 60,0 & 25 & 73,5 \\
\hline Noite & 23 & 40,0 & 9 & 39,1 \\
\hline
\end{tabular}


Dentre os 34 (59,6\%) profissionais da equipe de Enfermagem que relataram algum tipo de acidente de trabalho, no período de 2002 a 2011, 90\% deles afirmaram ter sofrido acidente de natureza biológica, 15\% deles julgaram-no sem risco eminente de contaminação, por terem perfurado o dedo com material estéril.

Entre as variáveis sociodemográficas avaliadas, houve correlação entre faixa etária, tempo de trabalho na categoria e a referência a acidentes ocupacionais, determinado pelo $\mathrm{p}<0,05$ (Tabela 2). A faixa etária mais jovem e aqueles com menos tempo de experiência na categoria apresentaram maior associação aos acidentes. Para as demais variáveis não houve associação estatisticamente significativa.

Avaliou-se a conduta imediata desse profissional após a ocorrência do acidente com risco biológico: lavaram a área exposta com água e sabão (73,5\%/25), lavaram o local com solução fisiológica $(8,8 \% / 3)$, não realizaram qualquer conduta $(8,8 \% / 3)$ e lavaram com antiséptico $(8,8 \% / 3)$.

Dos profissionais acidentados, 34\% (11) não notificaram os Acidentes de Trabalho, apontando mais de um motivo para a não execução da notificação: por falta de tempo (55\%), por não achar importante $(36 \%)$ e por não conhecer o instrumento de notificação - Comunicado de Acidente de Trabalho/CAT (9\%).

Tabela 2 - Associação estatisticamente significativa $(p<0,05)$ entre dados sociodemográficos e a ocorrência ou não de acidentes de trabalho. Arcos-MG, 2012

\begin{tabular}{|c|c|c|}
\hline \multirow[t]{2}{*}{ Variáveis } & \multicolumn{2}{|c|}{$\begin{array}{c}\text { Profissionais - referência à } \\
\text { acidentes de } \\
\text { Trabalho }\end{array}$} \\
\hline & Qui-quadrado & $\mathrm{P}$ valor \\
\hline Faixa etária (anos) & 8,56 & 0,04 \\
\hline $\begin{array}{l}\text { Tempos de } \\
\text { trabalho na } \\
\text { categoria (anos) }\end{array}$ & 14,85 & 0,02 \\
\hline
\end{tabular}

\section{DISCUSSÃO}

Nesta pesquisa houve predominância de técnicos de enfermagem na equipe, corroborada com dados do Conselho Regional de Enfermagem de Minas Gerais que ocupam a categoria mais numerosa (90.277), seguido dos enfermeiros (41.517) e, posteriormente, os auxiliares $(34.431)^{(7)}$. Supõe- se que auxiliares de enfermagem vêm buscando ampliar a profissionalização, alcançando o título de técnico de enfermagem, justificando o menor quantitativo neste estudo.

A equipe de enfermagem foi constituída em sua maioria pelo sexo feminino $(91,2 \%)$ reafirmando a ocupação das mulheres na enfermagem, fato que acompanha a profissão, e pode ser confirmado pelos dados do Conselho Federal de Enfermagem (COFEn), apresentados em pesquisa realizada em 2010, sendo o quadro brasileiro composto por $87,24 \%$ de profissionais de enfermagem mulheres ${ }^{(8)}$.

Em relação aos acidentes ocupacionais obteve-se maior relato entre os mais jovens e com menos experiência na profissão. Os numerosos procedimentos que levam a manipulação de objetos perfurocortantes, o ritmo intenso de trabalho, o manuseio inadequado, e a falta de profissionais para execução das atividades contribui para a maior incidência de acidentes de trabalho com perfurocortantes naquele grupo ${ }^{(9-10)}$.

Apesar das precauções determinadas por agências de saúde nacional e internacional, como o Ministério da Saúde e o Centro de Prevenção e Controle de Doenças dos EUA (CDC) e padronizados pelas equipes de enfermagem, os riscos de acidentes sempre estão presentes, influenciados pelos fatores de estresse, sobrecarga de trabalho, agitação psicomotora do paciente e transgressão das normas de prevenção pelos profissionais $^{(11-13)}$.

Nesta pesquisa, 90\% dos acidentes laborais com a equipe de enfermagem foram de natureza biológica. Em outra pesquisa em um Centro de Terapia Intensiva com o mesmo caráter avaliativo, obteve-se um percentual de $67,6 \%$ dos trabalhadores de enfermagem sofrendo acidentes com material biológico, sustentando a estatística da ocorrência deste tipo de acidente em diversas instituições e apontando como maior risco na equipe de enfermagem. É importante enfocar que a exposição ocupacional a material biológico é citado como uma das questões mais proeminentes na área da saúde e na segurança ocupacional, pois a equipe de enfermagem realiza inúmeras intervenções com materiais perfurocortantes $^{(14)}$.

Dos que sofreram acidente, 15\% deles julgaram o acidente sem risco iminente de contaminação, por terem perfurado o dedo com 
material estéril, porém é importante considerar que, frequentemente, os trabalhadores de saúde realizam trabalho em turnos, manipulam artigos inseguros, bem como não utilizam EPI ou fazem uso inadequadamente. Desta forma, estão expostos aos riscos de contato com micro-organismos veiculados por sangue ou outros fluidos corpóreos potencialmente infectados, por ferimentos provocados por agulhas, objetos pontiagudos, contato com mucosa, por descontinuidade da pele ${ }^{(15)}$. $\mathrm{O}$ fato de alguns profissionais considerarem-se isentos de risco quando se acidentam com material estéril torna-se preocupante, pois evidencia um erro técnico no trabalho, podendo ocorrer em outra atividade com material contaminado ${ }^{(16)}$.

Apesar da ausência de associação estatística entre categoria profissional e acidentes, os técnicos de enfermagem foram aqueles que mais os relataram (85\%). Isto pode ser explicado por representarem a categoria profissional de maior predominância, estar mais expostos ao risco por permanecerem a maior parte do tempo na assistência direta aos pacientes e executarem vários procedimentos invasivos ${ }^{(16)}$.

Devido ao número elevado da manipulação com agulhas, os acidentes ocupacionais ocasionados por materiais perfurocortantes entre os trabalhadores de enfermagem são frequentes, representando prejuízos tanto para os trabalhadores como para a instituição. Isso remete às necessidades constantes de atenção ao problema, principalmente em adotar ações para reduzir o número de acidentes, direcionar medidas para a notificação dos ocorridos, melhorar o fluxo de encaminhamento pósacidente e acompanhamento de saúde do trabalhador ${ }^{(17)}$.

O turno diurno apresenta a predominância de acidentes (76,5\%), possivelmente por concentrar maior volume de procedimentos e cuidados diretos aos pacientes comparado com o período noturno ${ }^{(18)}$. O relato de acidentes em hospitais no turno diurno foi apresentado em outra pesquisa, com $80,54 \%{ }^{(19)}$.

A conduta imediata dos profissionais após a ocorrência do acidente com risco biológico foi a preconizada por agência de saúde nacional e internacional: lavagem com água corrente e sabão do local lesado e, apenas com água corrente, quando o local lesado for mucosa.
O uso de antiséptico não é contraindicado, porém não há evidências de que o seu uso ou a expressão do local reduza o risco de contaminação, contudo a utilização de soluções irritantes (éter, glutaraldeído, hipoclorito de sódio) é contraindicada. Dependendo da gravidade do acidente, avaliada de acordo com critérios individuais, são necessárias condutas mais específicas e que requerem certos limites de tempo para execução, razão por que é primordial a notificação imediata do acidente ${ }^{(20-21)}$. É importante que, posteriormente à lavagem do local, sejam feitas coletas de sangue para sorologias e demais exames que se façam necessários de acordo com a avaliação do acidente, confecção da CAT, procedimentos quimioprofiláticos quando necessários e acompanhamento de saúde do trabalhador acidentado ${ }^{(17)}$.

Neste estudo 34\% dos acidentados não notificaram o fato e os motivos apontados foram preocupantes por refletir falta de sensibilização do profissional para o risco. Em outro estudo realizado sobre os acidentes com a equipe de enfermagem em um hospital de Palmas-estado de Tocantins, prevalece a baixa notificação $(35,9 \%)$ ao setor de Segurança do Trabalho do hospital, percentual aproximado deste estudo, demonstrando a clara existência da subnotificação, explicação do pouco conhecimento dos funcionários de como proceder corretamente no momento de notificar, verificando-se uma falha no processo da informação e sensibilização da importância de notificar o acidente ${ }^{(16)}$.

Entre os profissionais de saúde, a adesão às recomendações de biossegurança, dentre elas a notificação, tem sido baixa e distinta, considerando-se algumas variáveis, como sexo, categoria profissional, turno de trabalho, tempo de experiência e formação profissional, mesmo apresentando conhecimento suficiente sobre o tema "Biossegurança"(22). Isso pode estar relacionado com vários aspectos do comportamento humano, incluindo a falsa percepção de um risco e a subestimação da responsabilidade individual na resolução ou minimização de um problema. Destaca-se que nem sempre essa adesão insuficiente refere-se à falta de conhecimento acerca dos perigos, mas à falta de incorporação deste conhecimento à prática ${ }^{(23)}$.

Considera-se acidente notificado, aquele em que o profissional acidentado preenche a 
Comunicação de Acidente de Trabalho (CAT). O protocolo para o atendimento a esses profissionais inclui, além da CAT, o atendimento em serviço especializado. A notificação de acidente de exposição a material biológico tem grande importância para o profissional acidentado e para a instituição, ficando-os respaldados legalmente. Além disso, pode-se obter melhor conhecimento desses acidentes, de suas causas e consequências, para assim elaborar medidas preventivas, para determinar o risco de infecção do paciente-fonte e do profissional e para a adoção de medidas pós-exposição para a prevenção da transmissão de hepatite B e HIV ${ }^{(24-25)}$.

As causas de subnotificação de acidentes do trabalho evidenciam desinformação ou sensibilização em relação aos riscos e aos aspectos epidemiológicos e jurídicos que envolvem este tipo de acidente no ambiente hospitalar, bem como a submissão dos trabalhadores às condições de trabalho impostas pelo empregador, quando exteriorizam a falta de tempo para notificar o acidente $^{(26)}$.

Mesmo que alguns trabalhadores demonstrem conhecimento quanto à importância de precauções padrão no momento de cuidar do outro, a adoção destas não ocorre regularmente, consequentemente ampliando o número de acidentes de trabalho, além de pouco domínio quanto aos procedimentos após acidente ${ }^{(17)}$.

\section{CONCLUSÃO}

A equipe de enfermagem participante do estudo foi composta em sua maioria por técnicos de enfermagem, mulheres, com faixa etária entre 30 a 39 anos, com tempo de trabalho na categoria menor ou igual a cinco anos e realizando atividades em turno diurno.

Houve predominância de exposição ocupacional a material biológico entre os profissionais de enfermagem por meio de acidentes com material perfurocortante, sendo que a idade e a experiência profissional podem ser fatores associados.

O fato de apenas $34 \%$ notificarem os acidentes é preocupante, considerando os riscos da aquisição de patógenos transmitidos pelo sangue, de uma possível subestimação das consequências pelos profissionais, fluxo de notificação não definido pela instituição de saúde e pouca assistência imediata aos profissionais. O principal motivo apontado pelos profissionais da equipe de enfermagem para não notificar o acidente foi não achar relevante seguido pela falta de tempo.

Percebe-se a necessidade de estratégias de enfrentamento da subnotificação como conscientização da gravidade pelos profissionais, rever a segurança no ambiente de trabalho, capacitar a equipe quanto à manipulação segura de materiais perfurocortantes, explicitar o fluxo de atenção imediata após acidentes.

\section{REFERÊNCIAS}

1. Associação Brasileira de Enfermagem (ABEn). Cartilha do trabalhador de enfermagem: saúde, segurança e boas condições de trabalho. Rio de Janeiro [Internet] 2006. [acesso em 20 out 2013]. Disponível: http:// bvsms.saude.gov.br/bvs/publicacoes/cartilha_aben.pdf

2. Ribeiro AECS, Crhristinne RM, Espíndula BM. Identificação dos riscos institucionais em profissionais de enfermagem. Rev. Eletr. Enferm. Nutr. CEEN. [Internet] 2010;1(1) [acesso em 13 nov 2013]. Disponível: http://www.cpgls.ucg.br/ArquivosUpload/1/File/V\%20 MOSTRA\%20DE\%20PRODUO\%20CIENTIFICA/ SAUDE/15-.pdf

3. Nishide VM, Benatti MCC, Alexandre NMC. Ocorrência de acidente do trabalho em uma unidade de terapia intensiva. Rev. Latino-Am. Enfermagem. [Internet] 2004;12(2) [acesso em 15 set 2012]. Disponível: http:// dx.doi.org/10.1590/S0104-11692004000200009

4. Giomo DB, Freitas CTF, Alves LA, Robazzi MLCC. Acidentes de trabalho, riscos ocupacionais e absenteísmo entre trabalhadores de enfermagem hospitalar. Rev. enferm. UERJ. [Internet] 2009; 17(1) [acesso em 17 nov 2013]. Disponível: http://www. facenf.uerj.br/v17n1/v17n1a05.pdf

5. Sindicato dos Trabalhadores em Telecomunicações no Estado de São Paulo [Internet]. São Paulo: CAT (Comunicação de Acidente de Trabalho); [acesso em 20 ago 2014]. Disponível: http://www.sintetel.org/ novo/duvida_detalhes.php?id=13

6. Brasil. Lei $n^{\circ}$ 8.213, de 24 de julho de 1991. Dispõe sobre os Planos de Benefícios da Previdência Social e dá outras providências. Diário Oficial da União, Brasília, 25 jul. 1991. Seção 1:2.

7. Conselho Regional de Enfermagem (COREn) [Internet]. Belo Horizonte: Conselho Regional de Enfermagem de Minas Gerais; [acesso em 30 jun 2014]. Inscrições ativas; [1 tela]. Disponível: http://www.corenmg.gov.br 
8. Albuquerque GL, Persegona MFM, Freire NP. Análise de dados dos profissionais de enfermagem existentes nos Conselhos Regionais. Brasília: Cofen. [Internet] 2011;1 [Acesso em 12 mar 2014]. Disponível: http://www.pluridoc.com/ Site/FrontOffice/default.aspx? module=Files / FileDescription $\& I D=6111 \&$ state $=F D$

9. Nowak NL, Campos GA, Borba EO, Ulbricht L, Neves EB. Fatores de risco para acidentes com materiais perfurocortantes. MundoSaúde(Online) [Internet] 2013;37 [Acesso em 28 jan 2015]. Disponível em: http://www. saocamilo-sp.br/pdf/mundo_saude/155558/A06.pdf

10. Chiodi MB, Marziale MHP, Mondadori RM, Robazzi MLCC. Acidentes registrados no Centro de Referência em Saúde do Trabalhador de Ribeirão Preto, São Paulo. Rev. gauch. enferm. [Internet] 2010;31 [acesso em 10 jan 2014]. Disponível: http://dx.doi.org/10.1590/S198314472010000200002

11. Oppermann CM, Pires LC. Manual de biossegurança para serviços de Saúde. Porto Alegre: PMPA/SMS/ CGVS. 2003.

12. Centers For Disease Control and Prevention (CDC). Updated U.S. Public Health Service guidelines for the management of occupational exposure to HBV, $\mathrm{HCV}$, and HIV and recommendations for postexposure prophylaxis. MMWR Recomm Rep. [Internet] 2005;54 [acesso em 11 jan 2014]. Disponível: http://www.cdc. gov/mmwr/Preview/mmwrhtml/rr5011a1.htm

13. Ministério da Saúde (BR). Secretaria de Atenção à Saúde. Departamento de Ações Programáticas Estratégicas. Exposição a materiais biológicos. Brasília; 2006.

14. Bonini AM, Zeviani CP, Facchin LT, Gir E, Canini SRMS. Exposição ocupacional dos profissionais de enfermagem de uma unidade de terapia intensiva a material biológico. REE [Internet] 2009;11(3) [acesso em 05 jan 2014]. Disponível: http://www.fen.ufg.br/ fen_revista/v11/n3/pdf/v11n3a25.pdf

15. Magagnini MAM, Ayres JA. Acidentes com material biológico: a realidade de uma instituição hospitalar do interior paulista. Reme, Rev. Min. Enferm. [Internet] 2009;13(1) [acesso em 15 fev 2014]. Disponível: http:// reme.org.br/artigo/detalhes/171

16. Machado MRM, Machado FA. Acidentes com material biológico em trabalhadores de enfermagem do Hospital Geral de Palmas (TO). Rev. bras. saúde ocup. [Internet] 2011;36(124) [acesso em: 08 ago 2014]. Disponível: http://dx.doi.org/10.1590/S030376572011000200011

17. Vieira M, Padilha MICS. O HIV e o trabalhador de enfermagem frente ao acidente com material perfurocortante. Rev. Esc. Enferm. USP. [Internet] 2008;42(4) [acesso em 12 ago 2014]. Disponível: http://
dx.doi.org/10.1590/S0080-62342008000400026

18. Ribeiro EJG, Shimizu HE. Acidentes de trabalho com trabalhadores de enfermagem. Rev. bras. enferm. [Internet] 2007;60(5) [acesso em $10 \mathrm{dez}$ 2013]. Disponível: http://dx.doi.org/10.1590/S003471672007000500010

19. Miranzi SSC, Gaspar AACS, Iwamoto HH, Miranzi MAS, Dziabas DC. Acidentes de trabalho entre os trabalhadores de uma universidade pública. Rev. bras. saúde ocup. [Internet] 2008;33(188) [acesso em 05 jan 2014]. Disponível: http://dx.doi.org/10.1590/S030376572008000200005

20. Siegel JD, Rhinehart E, Jackson M, Chiarello L, and the Healthcare Infection Control Practices Advisory Committee, 2007 Guideline for Isolation Precautions: Preventing Transmission of Infectious Agents in Healthcare Settings. [Internet] 2007 [acesso em 13 jan 2013]. Disponível: http://www.cdc.gov/ncidod/dhqp/ pdf/isolation 2007

21. Ministério da Saúde (BR). Manual de condutas em exposição ocupacional a material biológico. [Internet] 2004 [acesso em 06 jan 2013]. Disponível: http://www. bvsde.paho.org/bvsamat/condutas.pdf

22. Oliveira AC, Cardoso CS, Mascarenhas D. Intensive care unit professionals' knowledge and behavior related to the adoption of contact precautions. Rev Latino-Am Enferm. [Internet] 2009;17(5) [acesso em 11 nov 2013]. Disponível: http://dx.doi.org/10.1590/ S0104-11692009000500005

23. Askarian M, Aramesh K, Palenik CJ. Knowledge, attitude, and practice toward contact isolation precautions among medical students in Shiraz, Iran. Am. J. Infect. Control [Internet] 2006;34(9) [acesso em 05 ago 2014]. Disponível: http://www.ajicjournal.org/ article/S0196-6553(06)00141-6/fulltext

24. Alves AP, Ferreira MD, Prearo MF, Gir E, Canini SRMS. Subnotificação de acidentes ocupacionais com material biológico pela enfermagem no bloco cirúrgico. Rev. Eletr. Enferm. [Internet] 2013;15(2) [acesso em 06 fev 2014]. Disponível: http://dx.doi. org/10.5216/ree.v15i2.18554

25. Ministério da Previdência e Assistência Social (BR). Informe previdência social. [Internet] 2001;13(10) [acesso em 10 out 2013]. Disponível: http://www.previdencia. gov.br/arquivos/office/3_081014-104627-116.pdf

26. Gusmão GS, Oliveira AC, Gama CS. Acidente de trabalho com material biológico: análise da ocorrência e do registro. Cogitare enferm. [Internet] 2013;18(3) [acesso em 12 ago 2014]. Disponível: http://ojs.c3sl.ufpr.br/ojs2/index.php/cogitare/article/ view/33572/21070 